\title{
Short-term probabilistic
} earthquake risk assessment considering time-dependent $b$

\section{values}

\section{Journal Article}

Author(s):

Gulia, Laura; Tormann, Thessa; Wiemer, Stefan (D); Herrmann, Marcus (D); Seif, Stefanie

Publication date:

2016-02-16

Permanent link:

https://doi.org/10.3929/ethz-b-000113775

Rights / license:

$\underline{\text { In Copyright - Non-Commercial Use Permitted }}$

Originally published in:

Geophysical Research Letters 43(3), https://doi.org/10.1002/2015GL066686

Funding acknowledgement:

153931 - Risk Governance of Deep Geothermal and Hydro Energy (SNF) 


\section{Geophysical Research Letters}

\section{RESEARCH LETTER}

10.1002/2015GL066686

Key Points:

- L'Aquila's foreshocks show significantly

lower $b$ values than background

seismicity

- Using time-dependent $b$ values increases forecasted main shock probabilities

- Such probabilities surpass levels to retrospectively warrant short-term evacuation

Correspondence to:

L. Gulia,

Igulia@ethz.ch

\section{Citation:}

Gulia, L., T. Tormann, S. Wiemer, M. Herrmann, and S. Seif (2016), Short-term probabilistic earthquake risk assessment considering timedependent $b$ values, Geophys. Res. Lett., 43, doi:10.1002/2015GL066686.

Received 21 OCT 2015 Accepted 19 JAN 2016 Accepted article online 22 JAN 2016

(C2016. American Geophysical Union. All Rights Reserved.

\section{Short-term probabilistic earthquake risk assessment considering time-dependent $b$ values}

\author{
Laura Gulia ${ }^{1}$, Thessa Tormann ${ }^{1}$, Stefan Wiemer ${ }^{1}$, Marcus Herrmann ${ }^{1}$, and Stefanie Seif ${ }^{1}$ \\ ${ }^{1}$ Swiss Seismological Service, ETH Zurich, Zurich, Switzerland
}

\begin{abstract}
Laboratory experiments highlight a systematic $b$ value decrease during the stress increase period before failure, and some large natural events are known to show a precursory decrease in the $b$ value. However, short-term forecast models currently consider only the generic probability that an event can trigger subsequent seismicity in the near field. While the probability increase over a stationary Poissonian background is substantial, selected case studies have shown through cost-benefit analysis that the absolute main shock probability remains too low to warrant significant mitigation actions. We analyze the probabilities considering both changes in the seismicity rates and temporal changes in the $b$ value. The precursory $b$ value decrease in the 2009 L'Aquila case results in an additional fiftyfold probability increase for a M6.3 event. Translated into time-varying hazard and risk, these changes surpass the cost-benefit threshold for short-term evacuation.
\end{abstract}

\section{Introduction}

Earthquakes tend to cluster in space and time, forming earthquake sequences. While major ruptures are usually followed by significant numbers of aftershocks, some destructive events have also precursory activity associated, in hindsight called foreshocks. Since there is currently no reliable way to differentiate between a foreshock and a main shock before the main shock occurred, foreshocks cannot be used deterministically for earthquake forecasting purposes. Short-term forecast models such as Epidemic Type Aftershock Sequence (ETAS) [Ogata, 1988] or STEP [Gerstenberger et al., 2005] so far consider a generic probability that an occurring event might trigger subsequent seismicity in the near field. Each event is regarded as a potential foreshock, which might produce aftershocks. With typically a few percent probability, a subsequent event may be larger than the initiating one [Reasenberg and Jones, 1989, 1994]. The triggering likelihood decays approximately with 1/time, following an Omori-Utsu decay [Utsu, 1961; Utsu et al., 1995]. The probability increase for a significant earthquake to happen, as assessed from rate increases during foreshock sequences, has proven to be clearly detectable but too low to warrant evacuation actions in view of a cost-benefit approach [van Stiphout et al., 2010].

A famous example for this situation is the M6.3 L'Aquila earthquake, which hit Italy in 2009 following 3 months of intense foreshock activity, causing severe damage and 309 casualties. While there was no scientific basis to foresee the impending main shock deterministically, the rate increase from the foreshock activity significantly increased the daily probability of a large event. van Stiphout et al. [2010] showed that this probability increase is not sufficient to reach the considered action levels in a risk analysis and cost-benefit approach for possible mitigation actions, i.e., in this case evacuation of vulnerable buildings.

Observations from laboratory experiments measuring acoustic emissions during loading cycles in pressurized rock samples have suggested that small events in the precursory phase of an impending large event change in their relative size distribution [Meredith et al., 1990; Main, 1996; Goebel et al., 2012]. They show an increasing relative proportion of larger events as the system approaches failure. This observation can be mathematically described by the $b$ value of the Gutenberg-Richter (GR) [1944] law, which estimates the number, $N$, of earthquakes larger or equal to magnitude, $M$, as $\log (N)=a-b M$. While the $a$ value is a measure of activity rate, the $b$ value describes the slope of the distribution of magnitude frequencies on a log scale. A lower $b$ value describes a distribution with a higher proportion of larger magnitudes, and vice versa. Laboratory observations document a systematic $b$ value decrease during the stress increase period before the main event [e.g., Goebel et al., 2012].

Predictions of this behavior from numerical modeling [Kun et al., 2013] and repeated measurements in the laboratory have inspired studies of natural earthquakes that might document the same observation, ideally with a sense of real-time predictability. While a number of large earthquakes have convincingly been shown 
to have ruptured areas of distinctly low pre-main shock $b$ values [e.g., Tormann et al., 2012; Schorlemmer et al., 2005; Tormann et al., 2015; Schurr et al., 2014], robust patterns in the temporal evolution are more difficult to detect with confidence. One difficulty being the very different scales suggested for a precursory drop in $b$ value: from a decadal scale decrease documented in the Tohoku main slip area [Nanjo et al., 2012] to a sudden drop a few days before the L'Aquila main shock [Papadopoulos et al., 2010].

Much effort has been put into understanding the details of the L'Aquila 2009 sequence. Using different data sets, De Gori et al. [2012] and Sugan et al. [2014] mapped the lowest along-fault $b$ values close to the small main shock nucleation region.

We investigate whether such observations can contribute to improving probabilistic short-term forecasting models and help decision makers. The 2009 M6.3 L'Aquila event offers the chance to study in detail both the foreshock signal in space (migration toward the epicenter) and time ( $b$ value decrease and rate increase) in the days before the main shock. Based on an improved catalog [Gasperini et al., 2013], we confirm the results first described by Papadopoulos et al. [2010] and then translate the time-dependent $a$ and $b$ values into daily probabilities of $M 6.3+$ events. We finally calculate the time-dependent risk, showing the impact of time-dependent GR parameters on a cost-benefit analysis.

\section{Data}

Meaningful analysis of $b$ value variation in time requires high-quality and consistent data: a homogeneous earthquake catalog for Italy in terms of moment magnitudes $\left(M_{w}\right)$ was assembled by Gasperini et al. [2013], who used event information from different available local magnitude catalogs for Italy (CSTI 1.1; CSI 1.1; BSI; ISIDE) and calibrated them with a set of homogeneous $M_{w}$ using general orthogonal regressions. The $M_{w}$ reference data set was built combining $M_{w}$ estimates from several moment tensor catalogs. Since the Italian seismic network was reorganized and extended in 2005 and the quality of hypocentral locations was improved, we use events starting 16 April 2005 up to 20 May 2012. We verified this period of best data quality via an equalized plot of the time-magnitude distribution [Agnew, 2015].

The 6 April L'Aquila earthquake nucleated at $42.342^{\circ} \mathrm{N}$ and $13.38^{\circ} \mathrm{E}$ at a depth of $8.3 \mathrm{~km}$. Both location and focal mechanism (pure normal faulting) suggest that the event occurred on the Paganica fault, a 15-18 km long, SW dipping structure [Chiarabba et al., 2009]. Its NW-SE orientation is consistent with the extensional fault systems of the central Apennine (Figure 1a).

A foreshock sequence started 3 months before the main shock, activating a region of about $10 \mathrm{~km}$ length and culminating with an $M_{w} 4.3$ event on 30 March and an $M_{w} 4$ on 5 April, a few hours before the main shock and near its hypocenter. Since the beginning of 2009 , the seismicity migrated toward the main shock nucleation point and was concentrated within $3 \mathrm{~km}$ radius in the last week (Figure 1a).

The largest historical event that likely ruptured the same fault occurred in 1461 , also with $M_{w} 6.3$, as estimated from macroseismic intensity data [Gasperini et al., 1999, 2010]. Paleoseismological investigations [Cinti et al., 2011] recognized five distinct surface faulting earthquakes: the 2009 event, one earthquake consistent with the 1461 event, a third event 1000 A.D., and two oldest events in the intervals 760 B.C. to 670 A.D. and 2900-760 B.C., respectively.

To analyze the temporal evolution of $b$ values in the L'Aquila source region, we select events in a circular area of $20 \mathrm{~km}$ radius, proportional to the fault length, centered on the main shock. The data set contains no quarry blasts [Wiemer and Baer, 2000; Gulia, 2010]. We assess the magnitude of completeness $\left(M_{c}\right)$ by the maximum curvature criterion [Wiemer and Wyss, 2002; Mignan et al., 2011].

We characterize the following periods of this activation cycle along the Paganica fault: (1) the long-term lowproductivity background seismicity (16 April 2005 to 31 December 2008), (2) the accelerating foreshock sequence with an increasing relative proportion of larger magnitudes and leading to the main shock (1 January 2009-last event before main shock), (3) the initially very high aftershock rates with a higher proportion of small events ("AFT1": three months, starting 16 April to avoid common incompleteness issues during the first few days following the main shock), and (4) a slow but steady return toward background levels, which have not been fully reached within the considered three years ("AFT2: 1 January 2010 to 31 May 2012").

We find the pre-main shock catalog to be complete above $M_{w} 1.3$, thus containing 297 background events and 200 foreshocks for the Frequency Magnitude Distribution (FMD) analysis. We stopped with the last event 

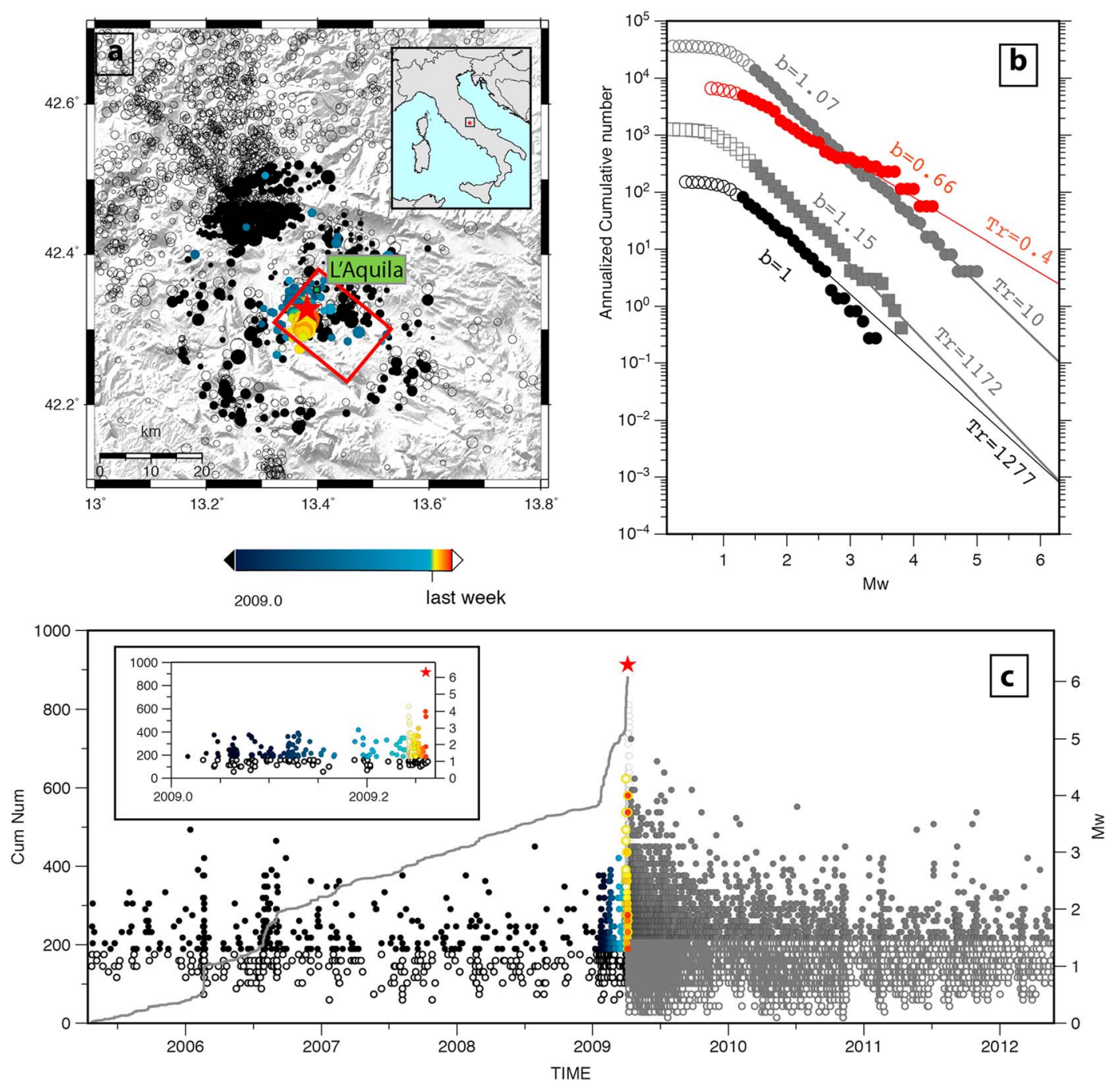

Figure 1. (a) Seismicity map showing background events (2005-2009, black circles) and foreshocks (color coded in time) in the $20 \mathrm{~km}$ radius study area centered on the epicenter (red star). Circles' sizes are proportional to $M_{w}$, spanning from 1.3 to 4.3. Black open circles: events outside the study area; red box: surface projection of the fault plane according to Serpelloni et al. [2012]. (b) FMDs of background events (black circles), last week of foreshocks (red circles), first 3 months of aftershocks after the first week (grey circles), and aftershocks from 2010 to May 2012 (grey squares). $T_{r}$ estimates (in years) are inferred for $M_{w} 6.3+$ events. (c) Plot of events in time: background (black), foreshocks (color coded), and aftershocks (grey). Open circles: events $<M_{c}$ grey line: cumulative number of all events.

before the main shock; i.e., our precursory signal is not driven by the occurrence of the major rupture itself. We estimate $M_{c}=1.5$ for the aftershocks, finding 3374 events for the AFT1 period and 691 events for the AFT2 period.

\section{Analysis and Results}

\subsection{Temporal $b$ Value Evolution}

Figure $1 \mathrm{~b}$ shows the annualized FMDs for the four distinct time periods discussed above. For each period we calculate the maximum likelihood $b$ value $[A k i, 1965]$ and estimate the expected recurrence time $\left(T_{r}\right)$ of an $M_{w} 6.3$ event from the annualized $a$ and $b$ values [Wiemer and Wyss, 1997].

The L'Aquila foreshock sequence is characterized not only by $\sim 10$ times higher rates of $M_{w} 1.3+$ events (as compared to the background activity) but also by a significant decrease of the $b$ value, which is reduced by more than $30 \%$ : from the background value $b_{\text {back }}=1.0 \pm 0.05$ to $b_{\mathrm{fs}}=0.66 \pm 0.05$ for events within the last week before the main shock. Those late foreshocks concentrated densely around the subsequent 

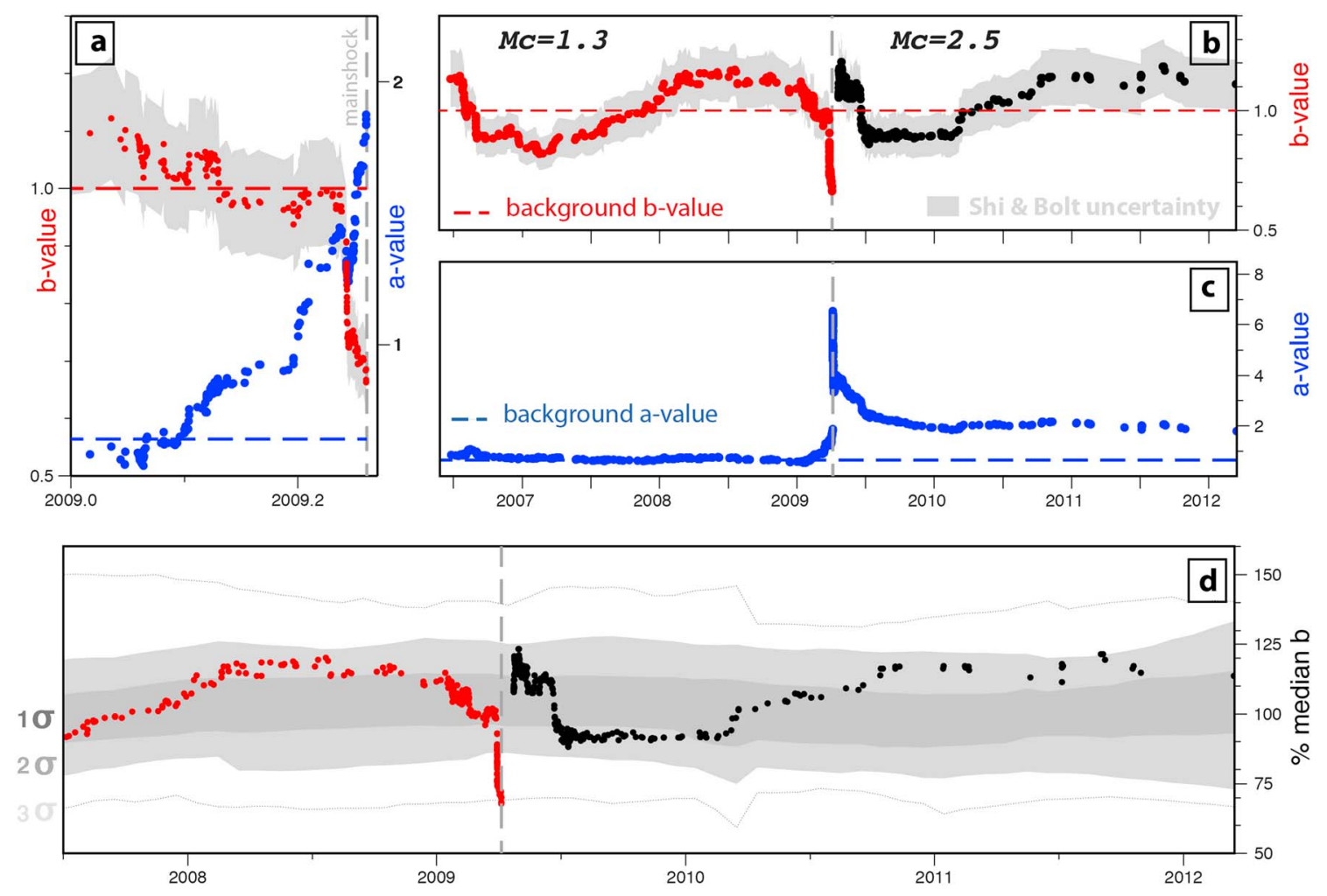

Figure 2. (a) Deviation of $a$ and $b$ values during foreshock period. (b) Temporal $b$ value evolution throughout the L'Aquila sequence. (c) Corresponding $a$ value evolution. (d) Significance of the L'Aquila pre-main shock (red) and post main shock (black) $b$ value variation, judged by the 1, 2, and 3 sigma levels of all contemporaneous local $b$ value variation throughout Italy.

nucleation point (Figures 1a and 1c). Aftershocks show an increased $b$ value, slightly $(\sim 10 \%)$ above $b_{\text {back. As }}$. A expected, activity rates are initially very high and decrease according to the Omori-Utsu law [Utsu et al., $1995]$ in the latest study period, then being back to 10 times the background rate of $M_{w} 1.3+$ events (Figure 1b). We note that the extrapolated rates of expected $M_{w} 6.3+$ events from the Gutenberg-Richter distribution increase by a factor of $>100$ between background and foreshocks, remain at similar levels throughout the early aftershock sequence, and return to background levels by the end of the study period (Figure 1c). The FMD comparison of the different periods meaningfully illustrates the characteristics of the different fault activation states but is only possible in retrospect, not during an ongoing seismic crisis.

To avoid artifacts in continuous $b$ value time series analysis, the choice of adequate techniques and parameter sensitivity assessment is crucial [Tormann et al., 2013; Kamer and Himer, 2013]. We use a window length of 100 events, which we move through the catalog event by event, thus exploring the full range of variability in the data. While this approach avoids the dependence on the choice of start time/event, we note that only every 100th data point is based on a fully independent data set. We feel that the nonindependence can be acknowledged more easily in the interpretation than random nonknowledge of potential variation that is introduced by longer step sizes between estimates.

We calculate maximum likelihood $b$ values $[A k i, 1965]$ and their standard uncertainty according to Shi and Bolt [1982], which is on the order of 10\% for the selected sample size [e.g., Tormann and Wiemer, 2014] (Figure 2b). We plot the values at the end of each time window to avoid confusion about causative relations: the $b$ value at each time step is purely based on past information. We find the $b$ value of the background seismicity to be rather stable, with some slow fluctuation of $\sim \pm 15 \%$ around the long-term mean, $b_{\text {back }}=1.0$ in this space-time window. However, with the accelerating foreshock activity (increasing $a$ values, Figure $2 \mathrm{c}$ ), the $b$ value continuously drops significantly, reaching its minimum of $0.66 \pm 0.05$ just before the main shock (Figure $2 \mathrm{~b}$ ). We verified that this signal is robust with respect to different window lengths within the reasonable range of 50-200 events. We assess the significance of this $b$ value drop with respect to temporal local $b$ value fluctuation measured from the seismicity observed throughout all of Italy during the same time period: on a $5 \mathrm{~km}$ 
spaced grid, we calculate local $b$ value time series from the closest 500 events (within a maximum radius of $50 \mathrm{~km}$ ). To avoid incompleteness issues after larger events, we stop a local data set whenever we detect an $M>5.5$ event within $100 \mathrm{~km}$ distance, thus using background and pre-main shock events. We use a general cutoff magnitude of 1.3 and assess the local completeness via maximum curvature, adding 0.2 [Woessner and Wiemer, 2005]. We calculate the local $M_{c}$ and scale the individual $b$ value time series by their median: the L'Aquila $b$ value drop is significant on a 3 sigma level (Figure $2 \mathrm{~d}$ ).

We extend the $b$ value time series analysis to the aftershock sequence, using the same window length but a fixed conservative $M_{c}=2.5$ excluding the events in the first week. The L'Aquila main shock productivity is the highest ever observed in Italy in the last 50 years, and the number of generated aftershocks is 3-10 times higher than for any previous earthquake of similar magnitude [Lolli et al., 2011]. The time evolution of the aftershock rates shows an unusual slow decay in the first 2 months, followed by a progressive acceleration and little sensitivity to the occurrence of strong aftershocks. Following an initial increase and strong variation, the aftershock $b$ values stabilize and return to similar fluctuations around the value of 1 , as already observed for the background activity; i.e., they remain largely within the 2 sigma level of average local variation (Figure $2 \mathrm{~d}$ ). The aftershock rates after 3 years reduced to $\sim 10$ times the background level, still slowly but steadily decreasing.

\subsection{The $\boldsymbol{b}$ Value-Dependent Foreshock Probabilities}

We compare five approaches to estimate the daily probability of main shock occurrence for this case study (Figure 3): three realizations of increasing complexity based on the Gutenberg-Richter model and two realizations of state-of-the-art ETAS models.

The most basic model ("Model GR1") is a long-term, time-independent background probability model, such as would be used in Poissonian probabilistic seismic hazard assessment: for the considered source region, a best estimate of background activity level ( $a$ value) and size distribution ( $b$ value) is calculated; the timeindependent probability for a target magnitude event derives directly from the Gutenberg-Richter law. Applied to our study area, we derive the parameter values $a_{\text {back_day }}=0.64$ and $b_{\text {back }}=1.0$ and estimate the daily background probability for an $M_{w} 6.3+$ event to be $\operatorname{Pr}_{\text {back }} 6.3+=0.002 \%$. Remarkably, the corresponding recurrence time (1200 years) is in agreement with the range of geological estimates (500-2000 years).

In "Model GR2," we use the same time-independent $b_{\text {back }}$ but estimate a time-varying $a$ value to account for rate increases observed during foreshock sequences: for each time step, we estimate the current $a$ value normalized by day from the most recent 100 events; i.e., the shorter the time period during which the latest 100 events were sampled, the higher the $a$ value. We update the model with each event in the catalog. The resulting probability curve follows the GR1 model through to the end of 2008 and then starts increasing until the main shock occurs, reaching a daily probability for an $M_{w} 6.3+$ event of $0.01 \%$.

In "Model GR3," we additionally include the temporal evolution of the $b$ value. That is, with each update, the current $a$ and $b$ values are estimated from the last 100 events and from the current probability for a target magnitude event. The resulting curve shows more variability, in particular, fluctuating by about \pm 1 order of magnitude around the GR1 estimate due to the fluctuations in local $b$ values during that time. With the onset of the foreshock sequence, the probability increases, at first very similar to Model GR2, but then it accelerates into a distinct peak reaching $\sim 0.45 \%$ daily probability for an $M_{w} 6.3+$ just prior to the main shock, when the $b$ values are lowest.

The most widely used forecast model in Operational Earthquake Forecasting is the Epidemic Type Aftershock Sequence (ETAS) [Ogata, 1988, 1998] model, which describes seismicity in time and space. It consists of a background and a triggering part, which combines the Gutenberg-Richter relationship, the modified Omori-Utsu law, and a function to describe the decay of aftershocks in space. The ETAS model characterizes the earthquake rate [events/(day $\left.\cdot \mathrm{km}^{2}\right)$ ] at a certain time and location through a normalized conditional intensity function. We estimate the ETAS parameters through maximum likelihood estimation [Zhuang et al., 2002] and distinguish two models: "ETAS1" uses the estimates of all parameters, whereas "ETAS2" uses a fixed value for $\alpha=\beta=b \cdot \log (10)$ to compensate for the incorrect assumption of isotropic aftershock distribution in space [Helmstetter et al., 2006; Hainzl et al., 2008a]. Target events, for which the log likelihood is optimized, are earthquakes which happen in a square of $200 \mathrm{~km}$ edge length centered on the main shock and within the time span: April 2005 to the last event before the main shock. Auxiliary events, which are used 


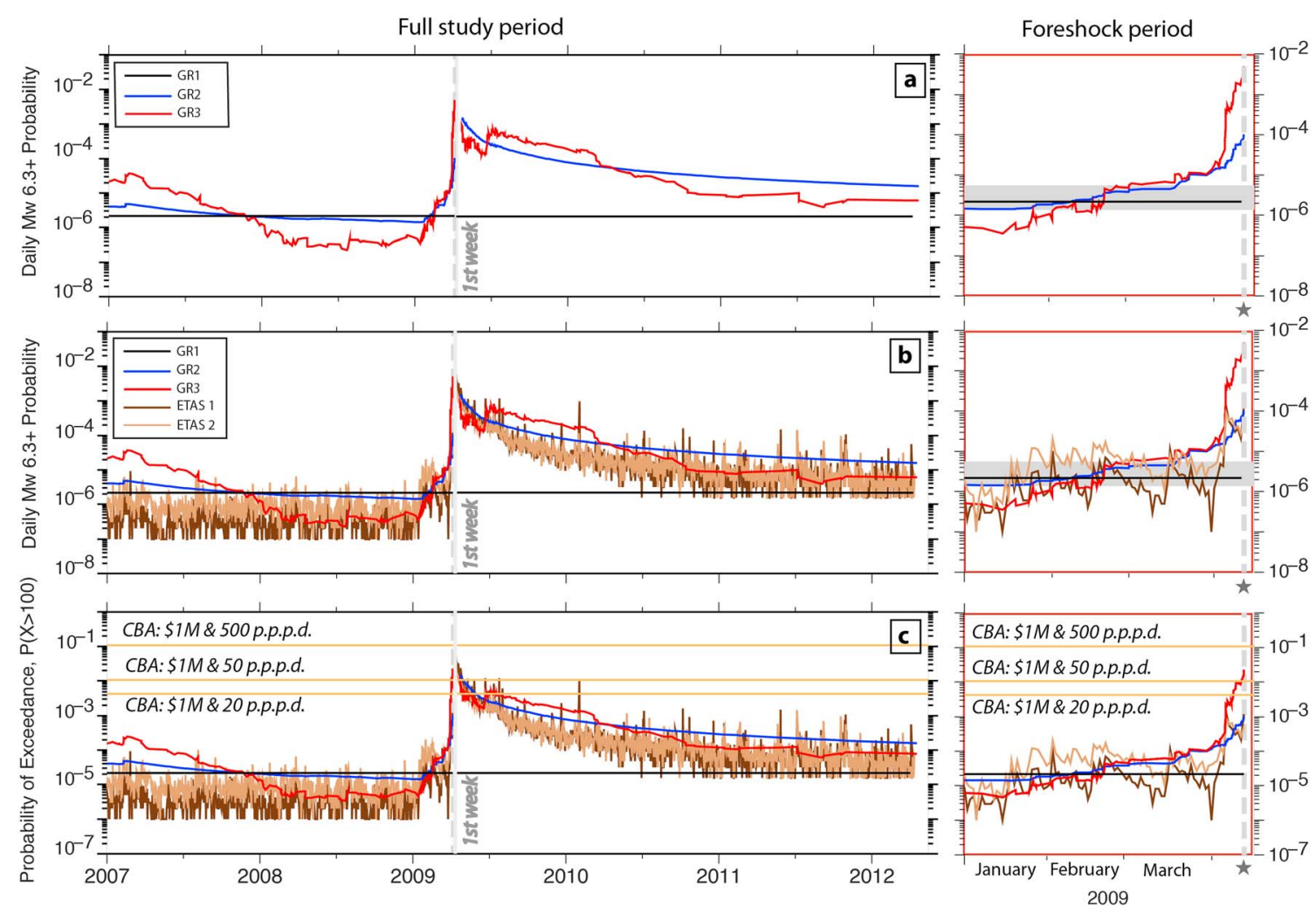

Figure 3. (a) Daily probabilities for $M_{w} 6.3+$ events estimated from three different realizations of the Gutenberg-Richter model: see text. Grey dashed line: 2009 main shock. First week of aftershocks (light grey bar) is not included in the calculation. (b) Same as Figure 3a, adding two realizations of the ETAS model ( $a$ fixed and $a$ free). (c) Probability of exceeding 100 fatalities in the next $24 \mathrm{~h}$ and selected CBA levels for evacuation of people (value of life $\$ 1 \mathrm{M}-\mathrm{evacuation}$ costs of $\$ 20$, $\$ 50$, and $\$ 500$ p.p.p.d) in vulnerable building. (right column) Corresponding details for foreshock period. Grey shading in Figures $3 \mathrm{a}$ and $3 \mathrm{~b}$ (right column) shows daily probability of $M 6.3$ events as derived from geological recurrence estimates.

as history, extend in space over a square of $400 \mathrm{~km}$ edge length and from 2000 to mid-April 2005. We perform 1 day forecasts for an $M_{w} 6.3+$, which estimate the number of earthquakes within the target region (radius $20 \mathrm{~km}$ ). The forecasts are realized through simulations, which incorporate the triggering effect of earthquakes from the auxiliary and target window up to the time before the forecast. We perform 100 simulations and calculate the mean. Just prior to the main shock, we observe $0.005 \%$ for ETAS1 and $0.01 \%$ for ETAS2 (Figure 3).

\subsection{The $\boldsymbol{b}$ Value-Dependent Risk Assessment Using Cost-Benefit Analysis}

We investigate whether the obtained probability increases over the long-term background might, in retrospect, justify mitigation actions during the L'Aquila seismic sequence. Analogue to the analysis proposed by van Stiphout et al. [2010], we translate forecast probabilities into time-varying seismic hazard and risk and apply a cost-benefit analysis (CBA) for evacuation actions at different costs (Figure $3 \mathrm{C}$ ). Within the variety of sensible mitigation actions [Jordan et al., 2014; Wang and Rogers, 2014], we restrict our analysis to the CBA quantifiable evacuation actions. CBA is a trade-off analysis where the expected costs for doing a mitigation action are minimized [Marzocchi and Woo, 2009]. If the expected loss exceeds the costs for an evacuation, then the benefit of evacuating is a monetary equivalent for saved human lives.

We first translate the earthquake rate forecasts into short-term hazard using the mean of four recent intensity predictions equations suitable for Italy [Bindi et al., 2011; Faenza and Michelini, 2010; Faccioli and Cauzzi, 2006; Allen et al., 2012]. We assume a site amplification of 0.45 intensity units. Since forecast rates and L'Aquila's exposure data are both represented as a single point location, we can simplify the hazard calculation-we locate the forecast rates beneath the city in a depth of $8.3 \mathrm{~km}$. As in van Stiphout et al. [2010], the loss estimations are based on QLARM, but we replace the casualty model with a model proposed by Zuccaro and Cacace [2011] for Italy and consider a constant building occupancy of $80 \%$. By combining the short-term hazard with 
the loss estimations, we can calculate the temporal evolution of risk throughout the sequence. We focus only on people within the most vulnerable building type: EMS-98 building type A (dry stones or clay, adobe or mud walls). We note that for the type $A$ buildings considered in this study and $80 \%$ occupancy rate, the two casualty models [Trendafiloski and Wyss, 2009; Zuccaro and Cacace, 2011] estimate almost identical risk; however, for lower occupancy rates or different building types the results differ significantly. We find that the probability gains from the GR3 model exceed predefined CBA action thresholds even before the main shock, suggesting evacuation of people living in vulnerable buildings. Specifically, 3 days before the main shock occurrence (Figure 3c), the risk for more than 100 fatalities in class A buildings exceeds evacuation costs of $\$ 50$ per person per day (p.p.p.d.) considering a value of a human life of $\$ 1 \mathrm{M}$.

\section{Discussion}

In this paper, we propose that including short-term-varying $b$ values into short-term risk models is sensible and may result in higher probability increases when compared to the long-term average hazard and risk estimates, as well as to the short-term clustering models of ETAS flavor currently used. Compared to those, the time-varying $b$ value GR3 model achieves, during the L'Aquila sequence, a probability increase for a M6.3 or larger event by a factor of 50. L'Aquila might be a unique example or it might be revealing a more general characteristic of some earthquake sequences. The latter would be consistent with observations of $b$ value decreases prior to the 2011 Tohoku [Nanjo et al., 2012] and 2014 lquique [Schurr et al., 2014] earthquakes. Our contribution, therefore, can be seen as another brick toward increasing the predictability of earthquakes (adopting the brick-by-brick analogy proposed by Jordan [2006]). We demonstrate that such gain is meaningful in the sense that it lifts the time-dependent risk curve out of the "insignificant for civil defense actions" into the realm of "actions should be considered," even talking about evacuation.

The beauty of the concept we propose for short-term risk assessment lies partially in its simplicity: it is a wellestablished fact that periods of increased seismic activity are also periods of increased seismic hazard and risk, because earthquakes cluster in space and time and are to a first order a scale-invariant process. Earthquake clustering models always win in formal testing by a large margin against time-independent models. We suggest that not only temporal changes in activity rates are indicative but also temporal changes in the earthquake size distribution, the $b$ value. This has been accepted for laboratory experiments for a long time, and ETAS and the Reasenberg-Jones models contain the $b$ value as a parameter, although in past studies it has been simply set to a regional average.

An obvious and critical unknown in time-dependent GR parameter analysis is whether observed changes are significant beyond "random" fluctuation. We could establish in this study that the L'Aquila $b$ value drop is highly significant in its strength compared to all Italian seismicity of the same time period. This certainly increases the confidence in the relevance of this type of analysis and our results shown in Figure 3, i.e., the increase in "real-time"-main shock probability and consequently the risk estimates reaching levels that would have suggested evacuation actions. However, this risk assessment remains a case study, and before a model such as GR3 could be even considered for assessing real-time risk in any kind of operational setting, it specifically needs to be tested in detail against many past sequences, including sequences that did not lead to a main shock. The model then needs to be tested in a fully prospective setup, such as the one proposed by the Collaboratory for the Study of Earthquake Predictability community (http://www.cseptesting.org/).

In addition to the temporal evolution of the $b$ value, there is a spatial signal: low $b$ values in the nucleation region mapped by De Gori et al. [2012] and Sugan et al. [2014], using different data sets and scales. The interpretation of the observed decrease in $b$ in terms of rupture initiation hence involves a spatial and temporal component, which are coupled to each other and, given the quantity and quality of data, cannot be unraveled uniquely. While it is feasible that the $b$ value of the rupture initiation region decreases in response to the increased shear stress, an alternative model suggests that the $b$ value decreases because the activity rate in an area of inherently lower $b$ value (i.e., a highly stressed patched of the fault) increases. This activity increase thus simply highlights a low $b$ value patch, resulting in an "apparent" transient in $b$. In our understanding, this interpretation offers the advantage that it is well known that relatively minor changes in stress level can result in large activity rate changes, while substantially changing the earthquake size distribution takes usually a dramatic change such as a main shock or volcanic intrusion [Wiemer and Wyss, 2000]. Following up this interpretation leads to an important hypothesis: foreshock probabilities are not generic 
but subject to significant spatial variability. During a swarm that activates a low $b$ value area, e.g., an asperity, they are considerably higher than during a swarm that occurs in a region of average $b$ values.

This study suggests that monitoring and assessing seismicity evolution should routinely consider potential changes in the relative size distribution of events. Apparently, microearthquakes are reflecting condictions (e.g., accumulated stress) that play a role for the likelihood of occurrence of larger events. A reliable measure of this requires sufficient seismic activity, which needs to be monitored with as low completeness level as possible and reported with consistent magnitudes.

\section{Conclusions}

1. We confirm the observation [Papadopoulos et al., 2010] that the decreasing $b$ value of the pre-main shock L'Aquila swarm reached unusually low values. We establish for the first time that this drop is significant on a 3 sigma level. Some previous studies classify the $b$ value decrease as deterministic precursor to the main shock; we interpret it as probabilistic indicator which produces a significant probability increase for a large event in the Gutenberg-Richter recurrence time extrapolation.

2. We translate the time-dependent $a$ and $b$ values into daily main shock probabilities, showing how they together result in daily probabilities of up to $0.45 \%$ just preceding the main shock. These values are a factor of $>1000$ above the background, and a factor of $\sim 50$ higher than the forecasted rates by the model currently considered state of the art in time-dependent forecasting. The result still implies that with more than $99 \%$ chance no such event will occur that day.

3. We improved the approach by van Stiphout et al. [2010], using more modern ground motion prediction equations, their epistemic uncertainty, and more suitable collapse matrices.

4. We show that L'Aquila's time-dependent risk, when considering time-dependent $b$ values, is a factor of 50 times higher than the risk using state-of-the-art time-dependent models. Assessed via CBA, this increase makes the difference to exceed the threshold for evacuation.

In our view probabilistic time-dependent risk assessment considering time-dependent $b$ values is a promising new concept that deserves to be studied more extensively in the future. Furthermore, we show that CBA is a reasonable way of investigating the potential impact of a model's probability gain.

\section{Acknowledgments}

We are grateful to Paolo Gasperini, Barbara Lolli, and Gianfranco Vannucci for providing the catalog and to Carlo Cauzzi for advising us in selecting appropriate attenuation relations. We thank the Editor and Andrew Michael, Jiancang Zhuang, and two anonymous reviewers for constructive reviews of the manuscript. The catalog data can be requested from Gianfranco Vannucci (gianfranco.vannucci@ingv.it) and the building vulnerabilities as well as MATLAB codes to compute $b$ value, time-dependent probabilities, and risk from Igulia@ethz.ch. Marcus Herrmann acknowledges support from the Swiss National Science Foundation, project 407040_153931. Stephanie Seif acknowledges support by ETH project 29 21-2.

\section{References}

Agnew, D. C. (2015), Equalized plot scales for exploring seismicity data, Seismol. Res. Lett., 86, 1412-1423, doi:10.1785/0220150054.

Aki, K. (1965), Maximum likelihood estimate of $b$ in the formula $\log n=a-b m$ and its confidence limits, Bull. Earthquake Res. Inst. Univ. Tokyo, 43, 237-239.

Allen, T. I., D. J. Wald, and C. B. Worden (2012), Intensity attenuation for active crustal regions, J. Seismol., 16(3), 409-433, doi:10.1007/ s10950-012-9278-7.

Bindi, D., F. Pacor, L. Luzi, R. Puglia, M. Massa, G. Ameri, and R. Paolucci (2011), Ground motion prediction equations derived from the Italian strong motion database, Bull. Earthquake Eng., 9(6), 1899-1920, doi:10.1007/s10518-011-9313-z.

Chiarabba, C., et al. (2009), The 2009 L'Aquila (central Italy) $M_{w} 6.3$ earthquake: Mainshock and aftershocks, Geophys. Res. Lett., 36, L18308, doi:10.1029/2009GL039627.

Cinti, F. R., D. Pantosti, P. M. De Martini, S. Pucci, R. Civico, S. Pierdominici, L. Cucci, C. A. Brunori, S. Pinzi, and A. Patera (2011), Evidence for surface faulting events along the Paganica fault prior to the 6 April 2009 L'Aquila earthquake (central Italy), J. Geophys. Res., 116, B07308, doi:10.1029/2010JB007988.

De Gori, P., F. P. Lucente, A. M. Lombardi, C. Chiarabba, and C. Montuori (2012), Heterogeneities along the 2009 L'Aquila normal fault inferred by the $b$-value distribution, Geophys. Res. Lett., 39, L15304, doi:10.1029/2012GL052822.

Faccioli, E., and C. Cauzzi (2006), Macroseismic intensities for seismic scenarios, estimated from instrumentally based correlations, in Proc. First European Conference on Earthquake Engineering and Seismology, pp. 1-10, Geneva, Switzerland, 3-8 Sept.

Faenza, L., and A. Michelini (2010), Regression analysis of MCS intensity and ground motion parameters in Italy and its application in ShakeMap, Geophys. J. Int., 180(3), 1138-1152, doi:10.1111/j.1365-246X.2009.04467.x.

Gasperini, P., F. Bernardini, G. Valensise, and E. Boschi (1999), Defining seismogenic sources from historical earthquake felt reports, Bull. Seismol. Soc. Am., 89, 94-110.

Gasperini, P., G. Vannucci, D. Tripone, and E. Boschi (2010), The location and sizing of historical earthquakes using the attenuation of macroseismic intensity with distance, Bull. Seismol. Soc. Am., 100(5A), 2035-2066, doi:10.1785/0120090330.

Gasperini, P., B. Lolli, and G. Vannucci (2013), Empirical calibration of local magnitude data sets versus moment magnitude in Italy, Bull. Seismol. Soc. Am., 103(4), 2227-2246, doi:10.1785/0120120356.

Gerstenberger, M. C., S. Wiemer, L. M. Jones, and P. A. Reasenberg (2005), Real-time forecasts of tomorrow's earthquakes in California, Nature, 96, 328-331, doi:10.1038/nature03622.

Goebel, T. H. W., T. W. Becker, D. Schorlemmer, S. Stanchits, C. Sammis, E. Rybacki, and G. Dresen (2012), Identifying fault heterogeneity through mapping spatial anomalies in acoustic emission statistics, J. Geophys. Res., 117, B03310, doi:10.1029/2011JB008763.

Gulia, L. (2010), Detection of quarry and mine blast contamination in European regional catalogues, Nat. Hazards, 53(2), 229-249, doi:10.1007/s11069-009-9426-8.

Gutenberg, B., and C. F. Richter (1944), Frequency of earthquakes in California, Bull. Seismol. Soc. Am., 34, 185-188. 
Hainzl, S., A. Christophersen, and B. Enescu (2008a), Impact of earthquake rupture extensions on parameter estimations of point-process models, Bull. Seismol. Soc. Am., 98(4), 2066-2072, doi:10.1785/0120070256.

Helmstetter, A., Y. Y. Kagan, and D. D. Jackson (2006), Comparison of short-term and time-independent earthquake forecast models for Southern California, Bull. Seismol. Soc. Am., 96(1), 90-106, doi:10.1785/0120050067.

Jordan, T. H. (2006), Earthquake predictability, brick by brick, Seismol. Res. Lett., 77, 3-7.

Jordan, T. H., W. Marzocchi, A. J. Michael, and M. C. Gerstenberger (2014), Operational earthquake forecasting can enhance earthquake preparedness, Seismol. Res. Lett., 85(5), 955-959.

Kamer, Y., and S. Himer (2013), Comment on "Analysis of the $b$-value before and after the 23 October $2011 M_{w} 7.2$ Van-Erciș, Turkey earthquake", Tectonophysics, 608, 1448-1451.

Kun, F., I. Varga, S. Lennartz-Sassinek, and I. G. Main (2013), Approach to failure in porous granular materials under compression, Phys. Rev. E, 88, 062207.

Lolli, B., P. Gasperini, and E. Boschi (2011), Time variations of aftershock decay of the 2009 April 6 L'Aquila (central Italy) earthquake: Evidence of the emergence of a negative exponential regime superimposed to the power law, Geophys. J. Int., 185, 764-774.

Main, I. G. (1996), Statistical physics, seismogenesis, and seismic hazard, Rev. Geophys., 34, 433-462, doi:10.1029/96RG02808.

Marzocchi, W., and G. Woo (2009), Principles of volcanic risk metrics: Theory and the case study of Mount Vesuvius and Campi Flegrei, Italy, J. Geophys. Res., 114, B03213, doi:10.1029/2008JB005908.

Meredith, P. G., I. G. Main, and C. Jones (1990), Temporal variations in seismicity during quasi-static and dynamic rock failure, Tectonophysics, $175,249-268$.

Mignan, A., M. J. Werner, S. Wiemer, C.-C. Chen, and Y.-M. Wu (2011), Bayesian estimation of the spatially varying completeness magnitude of earthquake catalogs, Bull. Seismol. Soc. Am., 101(3), 1371-1385, doi:10.1785/0120100223.

Nanjo, K. Z., N. Hirata, K. Obara, and K. Kasahara (2012), Decade-scale decrease in b value prior to the M9-class 2011 Tohoku and 2004 Sumatra quakes, Geophys. Res. Lett., 39, L20304, doi:10.1029/2012GL052997.

Ogata, Y. (1988), Statistical models for earthquake occurrences and residual analysis for point processes, J. Am. Stat. Assoc., 83(401), 9-27.

Ogata, Y. (1998), Space-time point-process models for earthquake occurrences, Ann. Inst. Stat. Math., 50(2), 379-402, doi:10.1023/ A:1003403601725.

Papadopoulos, G. A., M. Charalampakis, A. Fokaefs, and G. Minadakis (2010), Strong foreshock signal preceding the L'Aquila (Italy) earthquake $\left(M_{w} 6.3\right)$ of 6 April 2009, Nat. Hazards Earth Syst. Sci., 10, 19-24.

Reasenberg, P. A., and L. M. Jones (1989), Earthquake hazard after a mainshock in California, Science, 243, 1173-1176.

Reasenberg, P. A., and L. M. Jones (1994), Earthquake aftershocks: Update, Science, 265, 1251-1252.

Schorlemmer, D., S. Wiemer, and M. Wyss (2005), Variations in earthquake-size distribution across different stress regimes, Nature, 437(7058), 539-542, doi:10.1038/nature04094.

Schurr, B., et al. (2014), Gradual unlocking of plate boundary controlled initiation of the 2014 lquique earthquake, Nature, 512, 299-302, doi:10.1038/nature13681

Serpelloni, E., L. Anderlini, and M. E. Belardinelli (2012), Fault geometry, coseismic-slip distribution and Coulomb stress change associated with the 2009 April 6, $M_{w} 6.3$, L'Aquila earthquake from inversion of GPS displacements, Geophys. J. Int., 188, 473-489.

Shi, Y., and B. A. Bolt (1982), The standard error of the magnitude-frequency $b$ value, Bull. Seismol. Soc. Am., 72, 1677-1687.

Sugan, M., A. Kato, H. Miyake, S. Nakagawa, and A. Vuan (2014), The preparatory phase of the 2009 M6.3 L'Aquila earthquake by improving the detection capability of low-magnitude foreshocks, Geophys. Res. Lett., 41, 6137-6144, doi:10.1002/2014GL061199.

Tormann, T., and S. Wiemer (2014), Reply to Comment by Kamer on "Systematic survey of highresolution $b$ value imaging along Californian faults: Inference on asperities", J. Geophys. Res. Solid Earth, 119, 5834-5837, doi:10.1002/2014JB011269.

Tormann, T., S. Wiemer, and J. Hardebeck (2012), Earthquake recurrence models fail when earthquakes fail to reset the stress field, Geophys. Res. Lett., 39, L18310, doi:10.1029/2012GL052913.

Tormann, T., S. Wiemer, S. Metzger, A. Michael, and J. Hardebeck (2013), Size distribution of Parkfield's microearthquakes reflects changes in surface creep rate, Geophys. J. Int., 193(3), 1474-1478, doi:10.1093/gji/ggt093.

Tormann, T., B. Enescu, J. Woessner, and S. Wiemer (2015), Randomness of megathrust earthquakes implied by rapid stress recovery after the Japan earthquake, Nat. Geosci., 8, 152-158, doi:10.1038/ngeo2343.

Trendafiloski, G., and M. Wyss (2009), Loss estimation module in the second generation software QLARM, in Second International Workshop on Disaster Casualties, Univ. of Cambridge, U. K., 15-16 June.

Utsu, T. (1961), A statistical study on the occurrence of aftershocks, Geophys. Mag., 30(4), 521-605.

Utsu, T., Y. Ogata, and R. S. Matsu'ura (1995), The centenary of the Omori formula for a decay law of aftershock activity, J. Phys. Earth, 43, 1-33. van Stiphout, T., S. Wiemer, and W. Marzocchi (2010), Are short-term evacuations warranted? Case of the 2009 L'Aquila earthquake, Geophys. Res. Lett., 37, L06306, doi:10.1029/2009GL042352.

Wang, K., and G. C. Rogers (2014), Earthquake preparedness should not fluctuate on a daily or weekly basis, Seismol. Res. Lett., 85, 569-571.

Wiemer, S., and M. Baer (2000), Mapping and removing quarry blast events from seismicity catalogs, Bull. Seismol. Soc. Am., 90(2), 525-530, doi:10.1785/0119990104.

Wiemer, S., and M. Wyss (1997), Mapping the frequency-magnitude distribution in asperities: An improved technique to calculate recurrence times?, J. Geophys. Res., 102, 15,115-15,128, doi:10.1029/97JB00726.

Wiemer, S., and M. Wyss (2000), Minimum Magnitude of completeness in earthquake catalogs: Examples from Alaska, the western US and Japan, Bull. Seismol. Soc. Am., 90, 859-869.

Wiemer, S., and M. Wyss (2002), Mapping spatial variability of the frequency-magnitude distribution of earthquakes, Adv. Geophys., 45, 259-302.

Woessner, J., and S. Wiemer (2005), Assessing the quality of earthquake catalogues: Estimating the magnitude of completeness and its uncertainty, Bull. Seismol. Soc. Am., 95(2), 684-698.

Zhuang, J., Y. Ogata, and D. Vere-Jones (2002), Stochastic declustering of space-time earthquake occurrences, J. Am. Stat. Assoc., 97(458), 369-380, doi:10.1198/016214502760046925.

Zuccaro, G., and F. Cacace (2011), Seismic casualty evaluation: The Italian model, an application to the L'Aquila 2009 event, in Human Casualties in Earthquakes, edited by R. Spence, E. So, and C. Scawthorn, pp. 171-184, Springer, Dordrecht, Netherlands, doi:10.1007/97890-481-9455-1. 